Aims Since the publication of the Lancet commission in global surgery (2015) the need for more timely, accessible and affordable surgical care in low- and middle-income countries (LMIC) has been increasingly recognised. There is a paucity of data to guide healthcare advocacy and resource allocation. This student paediatrics society has collaborated with UK paediatric surgeons to develop a novel research hub (OxPLORE). OxPLORE will investigate three paediatric solid tumours (Wilm's tumours, Neuroblastomas and Rhabdomyosarcomas) and report their incidence, management and post-operative outcomes in differently resourced settings visited by medical students on elective placements.

Methods Students are currently forming links with consultants in their host centres, prior to the first batch of elective placements in November 2017 - February 2018. Host consultants are pairing elective students with local trainees and medical students.

Data will be anonymously recorded on a standardised proforma, by elective students and their host collaborators. The primary outcome is survival (at post-operative day 28, date of death, or date last seen alive). Secondary outcomes include: patient demographics; tumour stage; pre-operative management and morbidity (Clavien-Dindo Classification); operative details; post-operative management and follow up.

Ethical approval is arranged for each centre at an institutional level. All collaborators will be named authors on resulting publications.

Results In November and December, the project supervisors will run the first data collection pilot in Tanzania, and a student participant will gather data in Rwanda. Further OxPLORE collaborations will follow in early 2018.

The data from our UK centre has already been gathered (from 2012-2017). These data will be analysed and compared to Tanzanian and Rwandan data to describe the differing experiences of paediatric solid tumours in these centres.

Conclusions

- Information generated from these collaborations will inform individual centres of which tumours represent a greater burden of disease, and act as an advocacy tool to be used to obtain funding for surgical resources.

- OxPLORE students will develop relationships with link institutions, which will be nurtured for future collaborations driven by local colleagues.

- Comparative data may help to advise best management of paediatric solid tumours in differently resourced settings globally.

\section{G291(P) UNINTENTIONAL CHILDHOOD INJURIES IN SUB SAHARAN AFRICA - A FOCUSSED LITERATURE REVIEW 2009-2015}

B Eder, D Magnus. The School of Social and Community Medicine, University of Bristol, Bristol, UK

\subsection{6/archdischild-2018-rcpch.283}

Aims Childhood injury disproportionately affects children in low and middle-income countries and Sub Saharan Africa has the highest death rate in the world from injury. Given the burden of childhood injury in Sub Saharan Africa the allocation of resources for public health interventions to address this problem are inadequate. This focussed literature review aimed to describe the burden of childhood injury in Sub
Saharan Africa and to explore the extent to which trauma registries or injury databases are being used by focussing on research conducted following the Report on Child Injury Prevention.

Methods A search protocol was designed using the key terms 'child* or paediatric or paediatric' AND 'injur* or trauma' AND 'unintentional or accident".' The literature was reviewed for all studies in Sub Saharan Africa as defined by the United Nations. The databases Medline, Pubmed and Web of Science were searched for papers in the English Language that have been published since 2009. Twenty eight papers were identified for full text review and thirteen papers met the inclusion criteria. Data were extracted from the papers and common themes discussed.

Results Six countries out of the fifty one in Sub Saharan Africa had published papers on childhood unintentional injury since 2009. A large degree of heterogeneity existed between the studies. There were significant differences in the proportion of unintentional injuries due to either falls or road traffic incidents depending on the location of the study. Burns were found to be most common in children under the age of 5 whilst boys in all studies were more at risk of injury. Children were more likely to be injured at home or on the streets as opposed to school.

Conclusion Childhood unintentional injury in Sub Saharan Africa is under researched. Greater efforts need to be made to carry out research at a local level in order for public health interventions to produce the most benefit. The widespread implementation of trauma registries will help this process.

\section{G292(P) ESTIMATES OF THE BURDEN OF GROUP B STREPTOCOCCAL DISEASE WORLDWIDE FOR PREGNANT WOMEN, STILLBIRTHS AND CHILDREN}

${ }^{1} \mathrm{AC}$ Seale, ${ }^{1} \mathrm{~F}$ Bianchi-Jassir, ${ }^{1} \mathrm{NJ}$ Russell, ${ }^{1} \mathrm{M}$ Kohli-Lynch, ${ }^{1,2} \mathrm{CJ}$ Tann, ${ }^{3} \mathrm{~J}$ Hall, ${ }^{4} \mathrm{~L}$ Madrid, ${ }^{1}$ JE Lawn. ${ }^{1}$ MARCH Centre, London School of Hygiene and Tropical Medicine, London, UK; ${ }^{2}$ Neonatal Medicine, University College London Hospital, London, UK; ${ }^{3}$ Institute for Women's Health, University College London, London, UK; ${ }^{4}$ IS Global, University of Barcelona, Barcelona, Spain; ${ }^{5}$ Bayor College of Medicine, Houston, Texas, USA

\subsection{6/archdischild-2018-rcpch.284}

Background We aimed to estimate, for the first time, the global burden of Group B Streptococcus (GBS), with regards to invasive disease in infants, as well as in pregnant and postpartum women, and fetal infection/stillbirth. Intrapartum antibiotic prophylaxis (IAP) is currently used for prevention of early onset infant disease in high-income contexts, but is difficult to implement globally, and may contribute to antimicrobial resistance. Maternal GBS vaccines are in development.

Methods For 2015 live births, we used data from systematic reviews and meta-analyses (presented in separate papers in this GBS supplement) and a compartmental model to estimate:

- exposure to maternal GBS colonisation,

- cases of infant invasive GBS disease,

- deaths, and

- disabilities.

We applied incidence or prevalence data to estimate cases of maternal and fetal infection/stillbirth, and infants with invasive GBS disease presenting with neonatal encephalopathy (NE). We applied risk ratios to estimate numbers of preterm births attributable to GBS. Uncertainty was also estimated. 